\title{
Tailoring the Spectral Properties of Layered Chiral Mid-Infrared Metamaterials
}

\author{
H. R. BARNARD, ${ }^{*}$ AND G. R. NASH
}

AFFILIATIONS

Natural Sciences, College of Engineering Mathematics and Physical Sciences, University of Exeter, EX4 4QF, United Kingdom. *Author to whom correspondence should be addressed [hb409@exeter.ac.uk]

\section{ABSTRACT}

The characteristics of four layer chiral metamaterials, optically active in the important mid-infrared region, have been investigated using simulations and experiments. Results show that the spectral response of the materials can be tailored, whilst preserving the magnitude of the circular dichroism, relative to standard double layer metamaterials. An analysis of the coupling in these four layer structures shows that they offer greater design freedom than might be expected from a simple consideration of double layer structures.

Artificial chiral materials, such as metamaterials, have been the subject of growing interest as they can be engineered to have large optical activities across the electromagnetic spectrum [1][12]. Circularly dichroic metamaterials, where there is a difference in the extinction coefficient between the two circular polarizations (CP), are also capable of producing strongly localized CP light fields [5], [7], [13]. This effective spatial concentration of light would be particularly important if achieved in the mid-infrared (mid-IR) region of the spectrum, as it could improve the sensitivity and resolution of vibrational circular dichroism spectroscopy, an essential tool for characterizing chiral molecules via their vibrational modes [14]-[16].

One important class of chiral metamaterial that can be designed to have optical activity from thenear- to the far-infrared are those based on double-layered metallic resonators, where the resonators in one layer are twisted relative to those in the second [17]-[19]. In this work, we explore the properties of four-layer chiral metamaterials, consisting of U-shaped metallic resonators [20]-[22], which have only been previously studied theoretically [23]. We demonstrate materials exhibiting circular dichroism (CD) in the mid-IR, and show that the extra degrees of freedom offered in their design is attractive for the engineering of optically active materials with specific spectral characteristics.

To help understand the characteristics of four layer materials, the properties of a double layered chiral structure (Fig. 1(a)) were first studied. Each layer consists of four gold U-shaped SRRs, arranged in C4 symmetry, with each individual resonator rotated $90^{\circ}$ relative to the one directly underneath, and with the two layers separated by $\mathrm{SiO}_{2}$ dielectric layer. The top layer (B) is the mirror image of the bottom layer (A), and this arrangement eliminates the polarization dependence of a single SRR, minimizes any linear birefringence, whilst maintaining the CD.To achieve a response in the mid-IR, the dimensions of the SRR were chosen as $w=100 \mathrm{~nm}, \mathrm{~s}=700 \mathrm{~nm}$ and $d=150 \mathrm{~nm}$, with a spacer layer and gold thickness of $120 \mathrm{~nm}$ and $50 \mathrm{~nm}$ respectively.

Simulations were carried out using the FDTD software Lumerical, with a single unit cell modelled with periodic boundary conditions in the $\mathrm{x}$ and $\mathrm{y}$-directions over a period of $1.85 \mu \mathrm{m}$, and a light source linearly polarized in the $y$-direction incident from the negative z-direction. Frequency dependent refractive indices of the gold and silicon were calculated using the

Conductive 3D Materials contained within Lumerical [24] with conductivities of $45 \times 10^{6}(\Omega \mathrm{m})^{-1}[25]$ and $10(\Omega \mathrm{m})^{-1}$, and values of high frequency permittivities of 1 and 11.7, for gold and silicon respectively (note that conducting silicon substrates were used in experiments). The frequency dependent permittivity of the $\mathrm{SiO}_{2}$ was taken from Palak [24]. To allow the resonances of the metamaterials to be clearly seen, the transmission spectra were normalized to the spectra obtained from gold wire squares (closing the $\mathrm{U}$ shape in each resonator, to remove its resonance) on the same underlying layers as the gold U-shaped resonators. This eliminates the frequency dependence of the transmission through the whole structure, including the gold, silicon dioxide and silicon substrates, leaving only the response of the resonators.

Fig. 1(b) shows the simulated transmittance of the double layer metamaterial. There are two distinct minima, $\omega^{-}=1580 \mathrm{~cm}^{-1}$ and $\omega^{+}=2260 \mathrm{~cm}^{-1}$, which are due to each U-shape resonator coupling directly with the resonator immediately above/below it (without this coupling, for example if the layers were misaligned, the single broader resonance of a single SRR would be observed). The size of this splitting has previously been attributed to the relative twist angle between two adjacent structures [23], [26], [27], and has been quantified using an effective parameter [20] and equivalent circuit [28] approach.

In order to gain a more intuitive physical insight into the origins of the coupling and observed spectral splitting, and to aid in the understanding of four-layer metamaterials, we use a qualitative model of the induced electric currents and subsequent magnetic dipole orientations to describe the characteristics of these metamaterials. The simulated electric field profiles in the $x-y$ plane at both resonant frequencies are shown in Fig. 2(a). Since the source is polarized in the y-direction, only the resonators whose arms are intersected by this radiation will be excited; the 'light-atoms'. The direction of current flow in these structures is superimposed in white. Although the absolute direction is arbitrary, it is assumed that at some instant in time the illuminating radiation polarization is pointing in the negative $y$ direction and so all currents in the light-atoms will flow along the center line of the metamaterial, in this same direction. Dark-atom resonators cannot be excited directly by the incoming radiation, but can be excited via magneto-electric coupling from the adjacent light-atom [1], [23]. At a frequency of $\omega^{-}$, the direction of 
current flow in the light and dark-atoms is the same as this is the lower energy/frequency configuration. Conversely, at $\omega^{+}$the current directions in adjacent meta-atoms will oppose each other, as this is the higher energy/frequency configuration. The electric current directions in the dark-atoms are shown in Fig. 2(a) in black. The result of the current flow is an induced electric dipole across the gap between the arms of each SRR. However, as there is a $90^{\circ}$ twist angle between two coupled resonators, it is has been shown previously that the electric dipole interaction between layers can be ignored [26].

In contrast, the generated magnetic dipoles point out of the plane of the metamaterial, and therefore couple in the z-direction. The direction of these induced magnetic dipoles, identified from the direction of current flow in each SRR, are shown schematically in Fig. 2(b), along with the magnetic field profiles. As with current flow, the absolute direction of the magnetic dipole is arbitrary; but it is the relative directions assigned to adjacent resonators that is important. We have assigned a clockwise current flow to denote a dipole pointing into the plane and an anti-clockwise current flow to denote a dipole pointing out of the plane. At a frequency of $\omega$ the magnetic dipoles induced in each meta-atom pair, consisting of resonators in the two layers one on top of the other, are aligned, as shown schematically in Fig 3(a) consistent with this being the lower energy configuration. In contrast at $\omega^{+}$, the dipoles in each pair are anti-aligned and point in opposite directions, consistent with this being the higher energy configuration.

The properties of a four-layer metamaterial, constructed of two $\mathrm{AB}$ metamaterials stacked on top of one another, were then explored. The simulated electric and magnetic fields were analyzed as before, and the subsequent coupling is shown schematically in Fig. 3(b). In the lowest energy configuration, all of the dipoles point in the same direction in each of the four quadrants, whereas in the other, higher energy configuration, each dipole is opposing the one in the layer above or below. These two configurations therefore lead to two resonances in the transmittance spectrum (Fig. 3(c)), at $1440 \mathrm{~cm}^{-1}$ and $2220 \mathrm{~cm}^{-1}$, similar to the double layer case. In comparison to the double layer case, the resonances are $100 \mathrm{~cm}^{-1}$ further apart, which is consistent with the increased number of anti-aligned magnetic dipoles (see fig 3(b)) in the high energy configuration of the four layer material.

Fig. 4(a) shows a different, but still chiral, two layer (labelled as $\mathrm{E}$ and F) configuration, where the center of the unit cell is translated by half a period. The simulated spectral transmittance of this unit cell is identical to that of the AB structure since the arrays are equivalent. However, following the same analysis as that for the AB structure, the induced magnetic dipoles (Fig. 4(b)) are now opposite to those in the $\mathrm{AB}$ case. The $\mathrm{EF}$ configuration therefore provides a new building block with which to create a different four-layer metamaterial, $\mathrm{ABEF}$, and the resulting magnetic dipole configurations are shown in Fig. 4(c). The lowest energy configuration no longer corresponds to all the dipoles being aligned, as in the $A B A B$ case, but instead there are a total of 4 anti-aligned dipole pairings. The higher energy configuration has a total of 8 anti-aligned dipoles, lower than the 12 opposing dipoles in the $\mathrm{ABAB}$ case. Two different energy configurations are therefore allowed compared to the ABAB structure.

This is confirmed in the simulated transmittance of the $\mathrm{ABEF}$ structure (Fig. 4(d)) where, in contrast to the ABAB case, the resonances appear closer together spectrally at $\omega^{-}=1820 \mathrm{~cm}^{-1}$ and $\omega^{+}=2180 \mathrm{~cm}^{-1}$. The difference between the two spectra is consistent with the different arrangement of magnetic dipoles in the $\mathrm{ABAB}$ and $\mathrm{ABEF}$ configurations, in contrast to a previous theoretical study on a single U-shape resonator stack, which suggested that all four energies can be accessed in one structure [23]. Here we show that for a four-layer stack only two energies can be accessed, not four, and a different layer configuration (ABEF) must be introduced to access the remaining two energies. To confirm the results of the simulations, $\mathrm{AB}, \mathrm{ABAB}$ and $\mathrm{ABEF}$ structures were fabricated on $\mathrm{Si} / \mathrm{SiO}_{2}$ substrates. Metallic resonator structures were defined using electron beam lithography and thermal evaporation of $5 / 50 \mathrm{~nm}$ of chromium/gold, with successive layers separated by $140 \mathrm{~nm}(+/-$ $20 \mathrm{~nm})$ thick sputtered $\mathrm{SiO}_{2}$. Dimensions of the SRR were $\mathrm{w}=100 \mathrm{~nm}, \mathrm{~s}=600 \mathrm{~nm}$ and $d=150 \mathrm{~nm}$. The side length $\mathrm{s}$ is 50 $100 \mathrm{~nm}$ shorter than in simulations, as confirmed by an SEM image of a completed device, due to imperfect development of the pattern. Markers on the substrate were used to ensure alignment of the layers. Transmission spectra were measured in a Fourier transform infrared spectrometer, with incident light on the device linearly polarized using a wire grid polarizer. Measured spectra were normalized to the same simulated background spectrum used to normalize the simulated transmittance, and the background $\mathrm{CO}_{2}$ absorption at $2400 \mathrm{~cm}^{-1}$ was removed. There is good agreement between the measured transmittance spectrum of the AB structure, Fig. 5(a), with the simulated spectrum (Fig. $1(\mathrm{~b})$ ), with two clear resonances at $1730 \mathrm{~cm}^{-1}$ and $2050 \mathrm{~cm}^{-1}$ labelled $\omega^{-}$and $\omega^{+}$respectively. As in the simulated data, $\omega^{-}$ exhibits a lower transmittance than $\omega^{+}$, indicating that it is the easier of the two resonances to excite. In addition, the simulated resonances appear further apart than the measured resonances. This indicates that the coupling is stronger in the simulated structures than in the fabricated materials which could, for example, be due to differing dielectric properties of the spacer layer. Fig. 5(b) shows the measured transmittance of the fourlayer $A B A B$ device, which has two minima at $1570 \mathrm{~cm}^{-1}$ and $1790 \mathrm{~cm}^{-1}$, consistent with the simulated spectra (figure 3(c)). As expected from the simulations, the measured transmittance of the four-layer ABEF metamaterial, shown in Fig. 5(c), is also markedly different to that of the $\mathrm{AB}$ and $\mathrm{ABAB}$ structures, and does not contain two easily distinguishable resonances. This is due to the different configuration of coupled magnetic dipoles, which leads to two new resonant modes, which are much closer in frequency and therefore overlap. This is consistent with the fact that the coupling appears stronger in simulations than in experiment. In experiment, two small overlapping minima exist at $1400 \mathrm{~cm}^{-1}$, and around $1670 \mathrm{~cm}^{-1}$.

Finally, Fig. 5(d-f) shows the measured CD for all three structures. Here a quarter wave plate was used to generate both left hand and right hand CP incident light, and the transmission through the materials then measured (measured raw spectra were normalized to the same background used for the simulations). The $\mathrm{CD}$ was calculated as the difference in absorbance for each of the hands of incident light $(\triangle A=A L-A R$, where $\mathrm{AL}$ and $\mathrm{AR}$ are the absorbance under illumination with LHC and RHC circularly polarized light respectively). In the twolayer $\mathrm{AB}$ metamaterial, there is a maximum $\mathrm{CD}$ of approximately $30 \%$, occurring at both resonant frequencies, but with opposite signs. This is consistent with the CD observed in a similar metamaterial designed for shorter wavelengths [21], As expected from the simple qualitative analysis discussed above, themeasured circular dichroism of the four-layer $A B A B$ structure (Fig. 5(e)) is very similar to the $C D$ obtained for the $A B$ structure, whereas the measured $\mathrm{CD}$ spectra for the ABEF metamaterial, Fig. 5(f), has shifted to lower frequencies, whist still maintaining high levels of $\mathrm{CD}$. The magnitude of the $\mathrm{CD}$ remains approximately the same in all three structures, as this is determined by the chirality of a coupled pair, which is the same for all three metamaterials. Further work is underway to 
investigate other factors that could affect the size of the $\mathrm{CD}$, such as mis-alignment between layers, as more layers are added. Although the aim of this work was not to optimize the circular dichroism, but rather gain insight into the characteristics of these multi-layer materials, the CD could be increased by optimization of the characteristics of each vertically coupled resonator pair, for example by increasing the vertical magnetic coupling between layers, by reducing the separation or optimizing resonator geometry. At the same time, adding more layers, however, does provide an important insight for engineering the spectral response of these metamaterials whilst maintaining high levels of CD.

The stacking of chiral metamaterial layers, optically active in the important mid-IR region of the spectrum, was investigated using simulations and experiments. The measured transmittance spectrum of a four-layer metamaterial, consisting of two double layers stacked one on top of the other, was very similar to that of a single two-layer structure. The measured transmittance of a second type of four-layer metamaterial, incorporating one new double layer structure, had two resonances much closer in frequency, reflecting the fact that different energy configurations can be excited in this structure. As expected, the magnitude of the measured circular dichroism was broadly the same in all three structures (approximately 30\%), as all three materials contain the same basic chiral building block. However, we show that the spectral position of the maxima/minima CD can be tailored by stacking different configurations of the chiral metamaterial, demonstrating the feasibility of designing materials for specific applications, such as vibrational circular dichroism spectroscopy.

\section{FUNDING}

The authors acknowledge financial support from the Engineering and Physical Sciences Research Council (EPSRC) of the United Kingdom, via the EPSRC Centre for Doctoral Training in Metamaterials (Grant No.EP/L015331/1).

\section{ACKNOWLEDGMENTS}

The authors acknowledge useful discussions with Isaac Luxmoore, Eleanor Barr and Huanling Zou.

\section{DATA AVAILABILITY}

The data that support the findings of this study are available from the corresponding author upon reasonable request.

\section{REFERENCES}

[1] B. Sun and Y. Yu, "Analysis of Circular Dichroism in Chiral Metamaterial at Terahertz Frequencies," J.

Phys. D. Appl. Phys., 52(2), 025105, (2019).

[2] G. Kenanakis, R. Zhao, A. Stavrinidis, G.

Konstantinidis, N. Katsarakis, M. Kafesaki, "Flexible

Chiral Metamaterials in the Terahertz Regime: a

Comparative Study of Various Designs," Opt. Mater.

Express, 2(12), 1702, (2012).

[3] M. Masyukov, A. Vozianova, A. Grebenchukov, K. Gubaidullina, A. Zaitsev, and M. Khodzitsky,
“Optically Tunable Terahertz Chiral Metasurface

Based on Multi-layered Graphene," Sci. Rep., 10(1)

$1-10,(2020)$.

[4] P. Fei, G. A. E. Vandenbosch, W. Guo, X. Wen, D. Xiong,

W. Hu, Q. Zheng, X. Chen, "Versatile Cross-

Polarization Conversion Chiral Metasurface for

Linear and Circular Polarizations," Adv. Opt. Mater.,

8(13), 2000194, (2020).

[5] D.-H. Kwon, D. H. Werner, and P. L. Werner, "Optical

Planar Chiral Metamaterial Designs for Strong

Circular Dichroism and Polarization Rotation," Opt.

Express, 16(16), 11802-11807, (2008).

[6] J. Zhou, D. R. Chowdhury, R. Zhao, A. K. Azad, H. T.

Chen, C. M. Soukoulis, A. J. Taylor, J. F. O'Hara,

“Terahertz Chiral Metamaterials with Giant and

Dynamically Tunable Optical Activity," Phys. Rev. B -

Condens. Matter Mater. Phys., 86(3), 035448, (2012).

[7] M. Schäferling, X. Yin, N. Engheta, and H. Giessen,

“Helical Plasmonic Nanostructures as Prototypical

Chiral Near-Field Sources," ACS Photonics, 1(6), 530

537, (2014).

[8] J. K. Gansel, M. Latzel, A. Frölich, J. Kaschke, M. Thiel, and M. Wegener, "Tapered Gold-helix Metamaterials as Improved Circular Polarizers," Appl. Phys. Lett. 100(10), 101109, (2012)

[9] T. Cao, C. Wei, L. Mao, and Y. Li, "Extrinsic 2D

Chirality: Giant Circular Conversion Dichroism from a Metal-dielectric-metal Square Array," Sci. Rep., 4(1), 7442, (2014).

[10] Y. Zhao, A. N. Askarpour, L. Sun, J. Shi, X. Li, and A.

Alù, "Chirality Detection of Enantiomers using Twisted Optical Metamaterials," Nat. Commun., 8(1), 1-8, (2017).

[11] X. Zang, H. Gong, Z. Li, J. Xie, Q. Cheng, L. Chen, A. P. 
Shkurinov, Y. Zhu, S. Zhuang, "Metasurface for Multi-

channel Terahertz Beam Splitters and Polarization

Rotators," Appl. Phys. Lett., 112(17), 171111, (2018).

[12] M. S. Mahmud, D. Rosenmann, D. A. Czaplewski, J.

Gao, and X. Yang, "Plasmon-phonon Coupling

Between mid-infrared Chiral Metasurfaces and

Molecular Vibrations," Opt. Express, 28(14), 21192,

(2020).

[13] Y. Zhao, A. Qing, Y. Meng, Z. Song, and C. Lin, "Dual-

band Circular Polarizer Based on Simultaneous

Anisotropy and Chirality in Planar Metamaterial," Sci.

Rep., 8(1), 1729, (2018).

[14] E. Burgueño-Tapia and P. Joseph-Nathan,

"Vibrational Circular Dichroism: Recent Advances for

the Assignment of the Absolute Configuration of

Natural Products," Nat. Prod. Commun., 12(5), 641-

651, (2017).

[15] X. Li, K. H. Hopmann, J. Hudecová, W. Stensen, J.

Novotná, M. Urbanová, J. S. Svendsen, P. Bouř, K.

Ruud, "Absolute Configuration of a Cyclic Dipeptide

Reflected in Vibrational Optical Activity: Ab initio

and Experimental Investigation," J. Phys. Chem. A,

116(10), 2554-2563, (2012).

[16] M. O. Okuom, R. Burks, C. Naylor, and A. E. Holmes,

“Applied Circular Dichroism: A Facile Spectroscopic

Tool for Configurational Assignment and

Determination of Enantiopurity," J. Anal. Methods

Chem., 2015(865605), (2015).

[17] S. Fasold, S. Linß, T. Kawde, M. Falkner, M. Decker, T.

Pertsch, I. Staude, “Disorder-Enabled Pure Chirality

in Bilayer Plasmonic Metasurfaces," ACS Photonics,

5(5), 1773-1778, (2018).

[18] Z. Y. Zhang, F. Fan, T. F. Li, Y. Y. Ji, and S. J. Chang,

“Terahertz Polarization Conversion and Sensing with
Double-Layer Chiral Metasurface," Chinese Phys. B,

29(7), 78707-078707, (2020).

[19] Y. Cheng, F. Chen, and H. Luo, "Multi-band Giant

Circular Dichroism Based on Conjugated Bilayer

Twisted-semicircle Nanostructure at Optical

Frequency," Phys. Lett. A, 384(19), 126398, (2020).

[20] Z. Li, R. Zhao, T. Koschny, M. Kafesaki, K. B. Alici, E.

Colak, H. Caglayan, E. Ozbay, C. M. Soukoulis, . “Chiral

Metamaterials with Negative Refractive Index Based

on Four 'u' Split Ring Resonators," Appl. Phys. Lett. 97(8), 081901, (2010).

[21] M. Decker, R. Zhao, C. M. Soukoulis, S. Linden, and M. Wegener, "Twisted Split-ring-Resonator Photonic

Metamaterial with Huge Optical Activity," Opt. Lett., 35(10), 1593, (2010).

[22] X. Xiong, W. H. Sun, Y. J. Bao, M. Wang, R. W. Peng, C. Sun, X. Lu, J. Shao, Z. F. Li, N. Ming, “Construction of a Chiral Metamaterial with a U-Shaped Resonator Assembly," Phys. Rev. B - Condens. Matter Mater. Phys., 81(7), (2010).

[23] N. Liu and H. Giessen, "Three-dimensional Optical Metamaterials as Model Systems for Longitudinal and Transverse Magnetic Coupling," Opt. Express, 16(26), 21233, (2008).

[24] "Lumerical FDTD Solutions.", https://www.lumerical.com/ [accessed 30th July 2017].

[25] K. Barbalace, "Kenneth Barbalace. Periodic Table of Elements. EnvironmentalChemistry.com.," 1995. [Online].. [Accessed: 30-Jul-2017].

[26] N. Liu, H. Liu, S. Zhu, and H. Giessen, "Stereometamaterials," Nat. Photonics, 3(3), 157162, (2009).

[27] N. Liu and H. Giessen, "Coupling Effects in Optical 
Metamaterials," Angewandte Chemie - International

Edition, 49(51). 9838-9852, (2010)

[28] E. Cubukcu, S. Zhang, Y. Park, G. Bartal, X. Zhang, S.

Zhang, "Split Ring Resonator Sensors for Infrared

Detection of Single Molecular Monolayers," Appl.

Phys. Lett., 95(4), 043113, (2009).

Figure 1 - Double layer AB metamaterial. (a) Device schematic of a double layered chiral metamaterial. Inset: SEM image of fabricated device (b) Normalised, simulated transmittance spectrum of an array of $\mathrm{AB}$ structures illuminated with a linearly polarised source. $\omega$-and $\omega^{+}$correspond to the low/ high frequency resonances respectively.

Figure 2 - Simulated electric and magnetic field profiles of AB metamaterial. (a) Simulated electric field profiles of AB structure at $\omega$ and $\omega^{+}$, for both layers of the device, under $y$-linearly polarized illumination. White and black arrows define direction of current flow for the light and dark-atoms respectively. (b) Simulated magnetic field profiles of AB structure at $\omega^{-}$and $\omega^{+}$, for both layers of metamaterial. Resultant magnetic dipole directions are shown as; a cross denoting the dipole pointing into the plane of the surface, and is the result of a clockwise rotation of current, whereas the dot points out of plane and is a result of an anti-clockwise current flow.

Figure 3 - Magnetic dipole configurations. (a) Summary of magnetic dipole orientations in the AB structure. (b) Schematic describing the two allowed energy states in the four layer ABAB metamaterial. The low-low and high-high configurations. (c) Simulated transmission spectrum of ABAB metamaterial under linearly polarized illumination. $\omega^{-}$and $\omega^{+}$correspond to the low and high frequency resonances respectively.

Figure 4 - Two layer EF metamaterial and subsequent four layer ABEF metamaterial. (a) Device schematic of double layered metamaterial, in which the unit cell has been shifted by half a period. Dimensions are the same as the AB device. (b) Schematic representation of the magnetic dipole orientations in the double layered EF metamaterial. (c) Schematic representation of the magnetic dipole orientations in an ABEF metamaterial. The low (high) energy configuration is formed by stacking the low (high) AB device with a low (high) EF device. (d) Simulated transmission spectra of the ABEF device under lineally polarized illumination. The low $\left(\omega^{-}\right)$and high $\left(\omega^{+}\right)$frequency resonances are marked on the graph.

Figure 5 - Experimental transmittance and circular dichroism (a), (b), (c) show the experimental transmittance of an $A B$, $A B A B$ and $A B E F$ metamaterial respectively. Measured with a linearly polarized source and normalized to a background. (d), (e), (f) the circular dichroism of each metamaterial. 


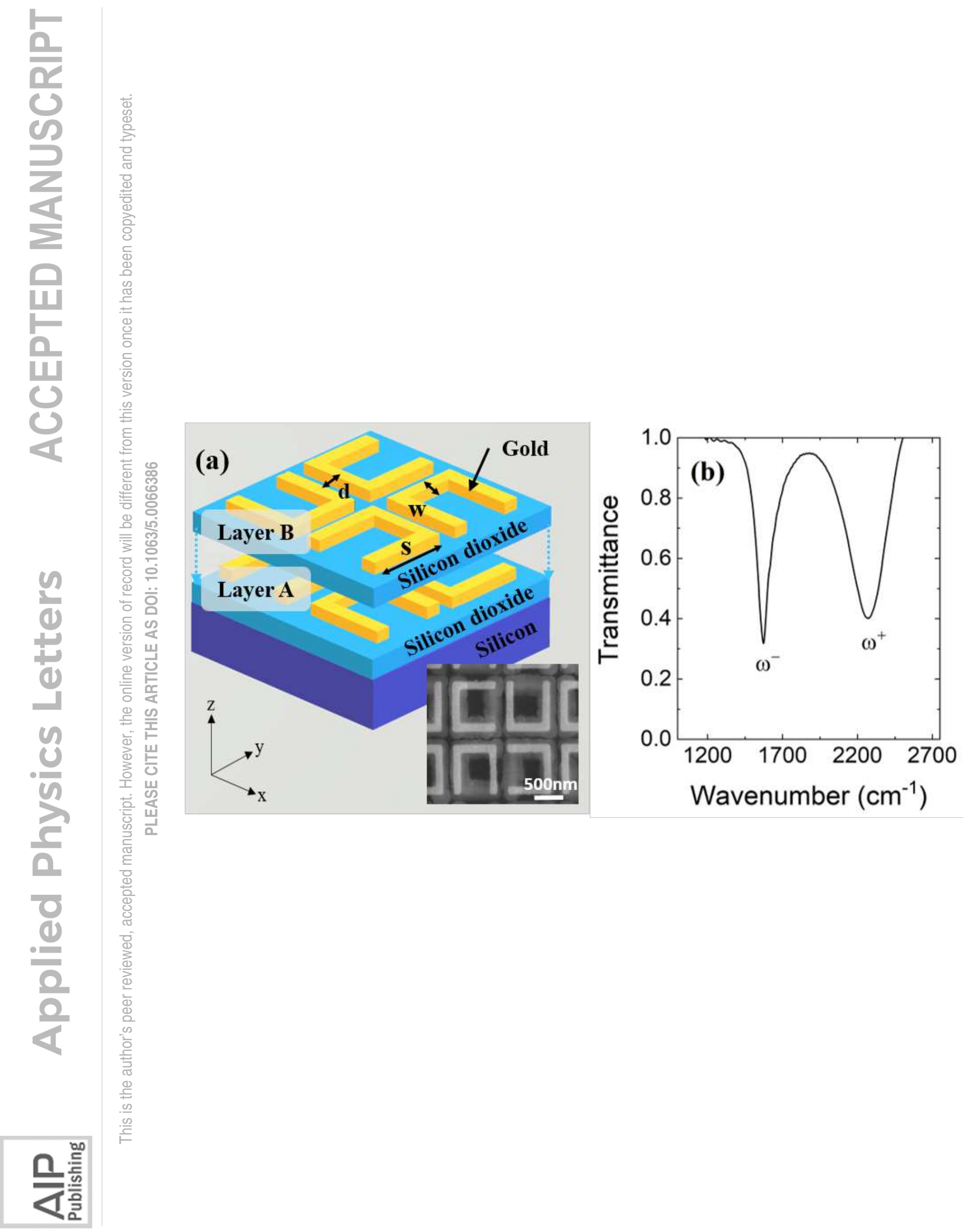




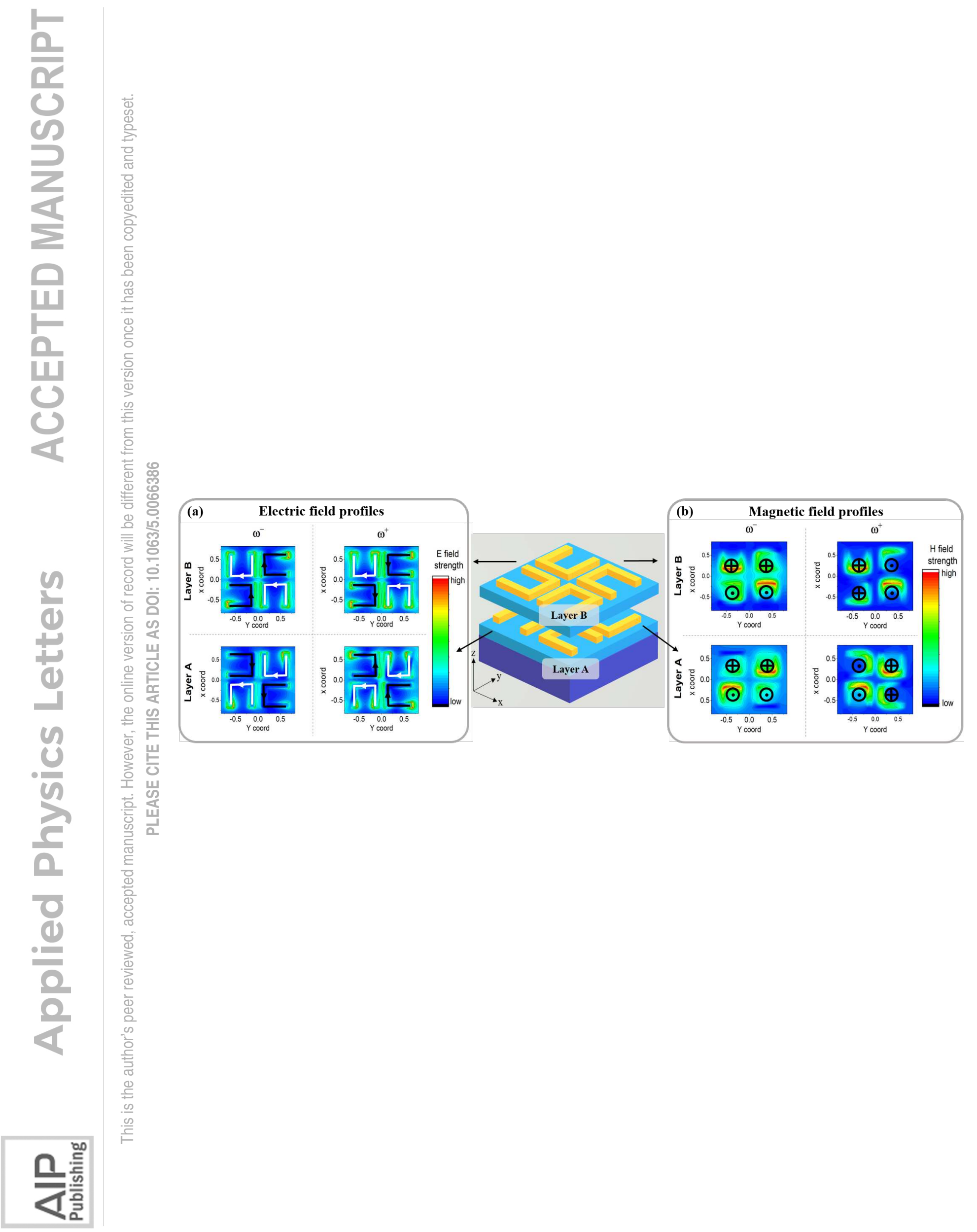



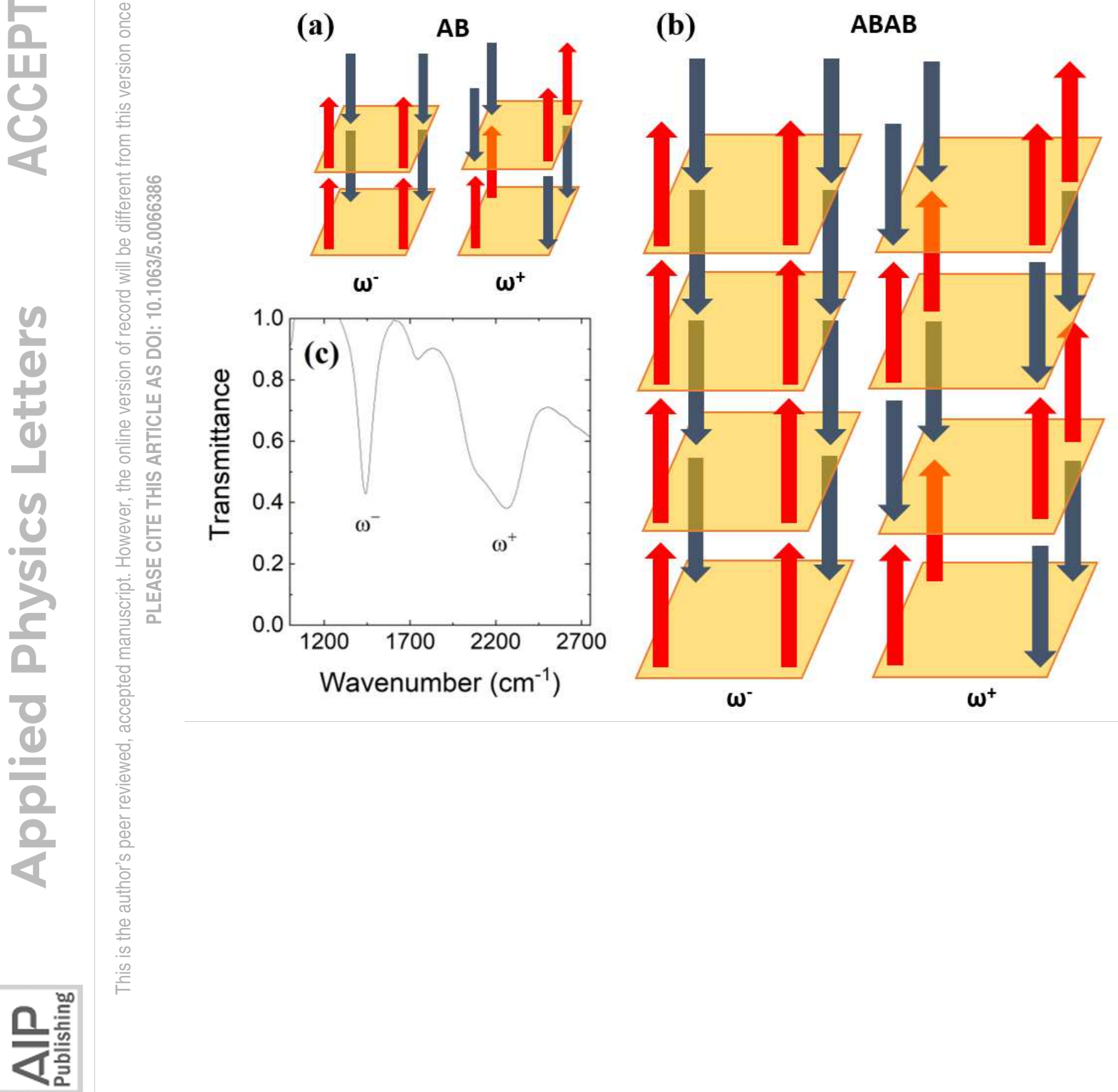

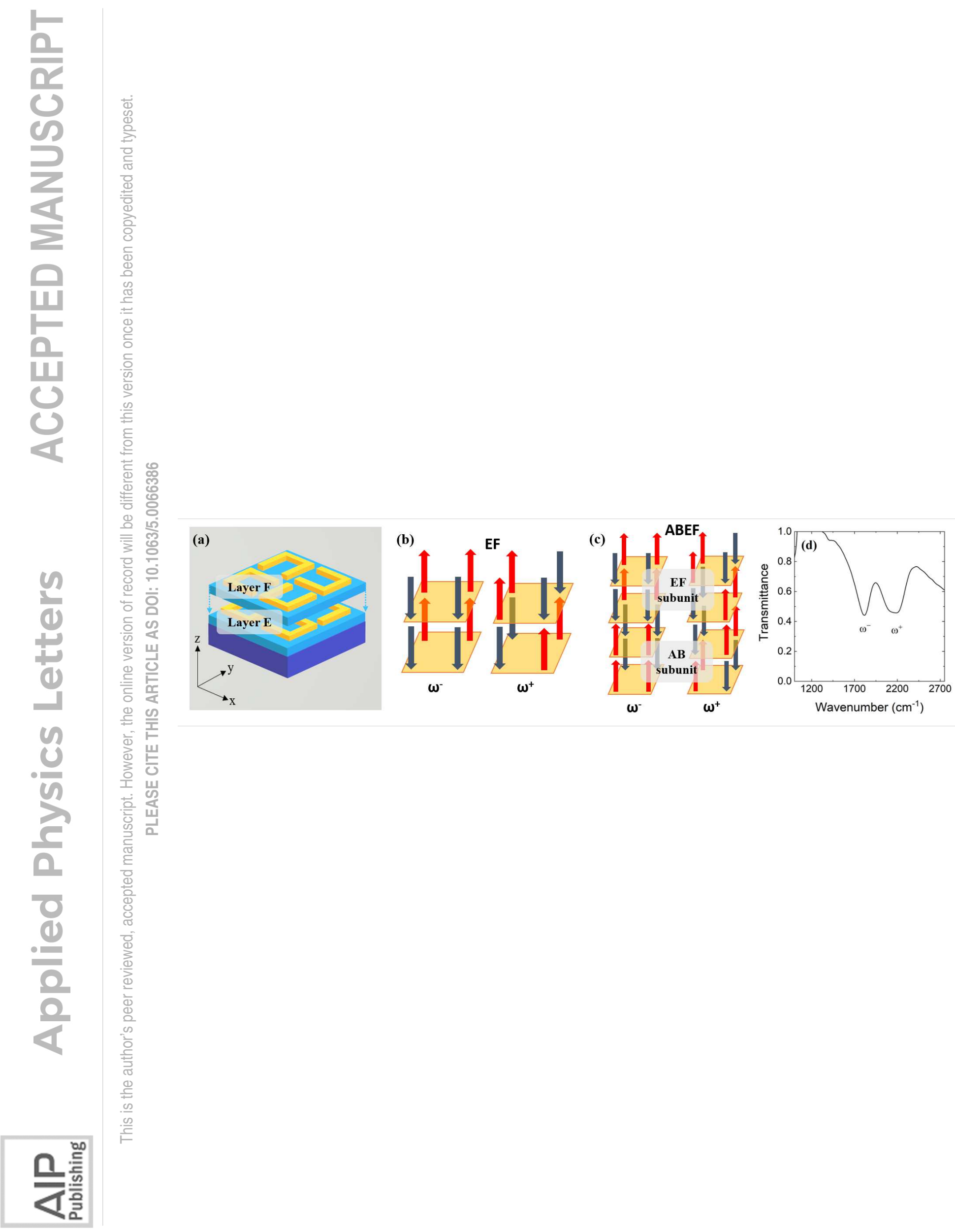


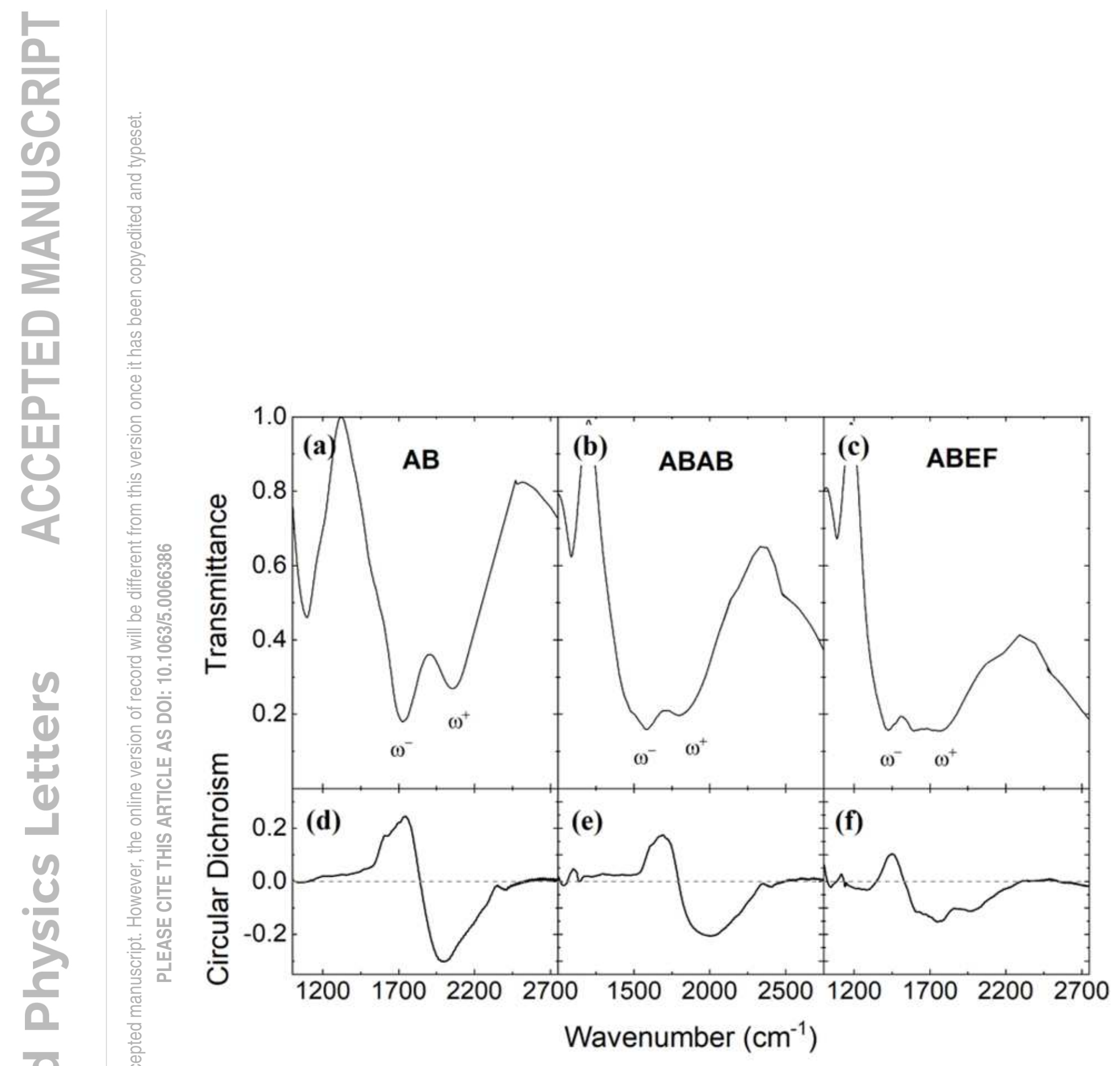

